\title{
The precedence of binocular fusion over binocular rivalry
}

\author{
RANDOLPH BLAKE and KARIN BOOTHROYD \\ Northwestern University, Evanston, Illinois
}

\begin{abstract}
During fusion, when both eyes view a set of identical vertical contours, reaction times (RT) to decrements in contrast of either set of contours are very brief. When the right eye views a set of vertical contours and the left eye views a set of horizontal contours, vigorous binocular rivalry results. RTs to decrements in the contrast of one set of contours is brief if those contours are dominant in rivalry; RT is lengthened considerably if those contours are suppressed. When the right eye views a set of vertical contours and the left eye views a set of horizontal contours and a set of vertical contours, RTs to decrements in contrast of any of those contours are comparable to those measured during fusion, not during rivalry. This pattern of results indicates that the presence of matching features in the two eyes' views renders those features exempt from binocular suppression; binocular fusion takes precedence over binocular rivalry.
\end{abstract}

The human visual system receives information about the layout of objects in the environment from two sources, the left and right eyes. If the information received by the two eyes is compatible, the brain combines their inputs in a way that yields a stable, unitary percept. This process of combination is often referred to as "binocular fusion." Working together in this fashion, the two eyes outperform either eye alone, providing an observer with enhanced visual sensitivity (Blake \& Fox, 1973), improved visuomotor coordination (Jones \& Lee, 1981), and keenly accurate depth information (Ogle, 1964). When, however, the two eyes receive incompatible information, stable single vision gives way to fluctuations in dominance between the two eyes (Breese, 1899). This breakdown in binocular combination, known as "binocular rivalry," renders one eye (or portions of that eye) temporarily less sensitive to visual information (e.g., Fox \& Check, 1968).

Over the years, there has been considerable interest in the relation between the processes of binocular fusion and binocular rivalry. For a time, some visual scientists believed that binocular rivalry was the only process involved in binocular single vision, with apparent fusion resulting from rivalry ongoing inconspicuously (e.g., Asher, 1953). This strict suppression theory is now largely rejected in favor of models that incorporate both fusion and suppression processes (e.g., Nelson, 1975). According to these models, fusion and rivalry can coexist within different parts of the visual field: objects situated on or near the horopter produce compatible monocular images that are seen as single, whereas objects located well off the horop-

This work was supported by a grant from the National Science Foundation (BNS82-00850). We thank Geoffrey Iverson and Steve Harp for help with the statistical analysis and Karen Holopigian, David Westendorf, and Walter Makous for comments on an earlier version of this paper.

The authors' mailing address is: Cresap Neuroscience Laboratory, Northwestern University, 2021 Sheridan Rd., Evanston, IL 60201. ter produce dichoptic images that result in suppression (Blake \& Camisa, 1978; Ono, Angus, \& Gregor, 1977). But what transpires when rivalry and fusion are pitted against one another within the same portion of the visual field? Which outcome, combination or suppression, takes precedence?

One way to think about this question is to consider the effects of rivalry suppression on stereopsis. Several investigators have studied this problem, but the results are not entirely unequivocal. Hochberg (1964) reported that when one eye's view is totally suppressed during rivalry, stereopsis is abolished. In his experiment, Hochberg had observers view stereograms consisting of two disparate circles. Superimposed on one half-image of the stereogram were horizontal lines. According to Hochberg's observers, all traces of stereoscopic depth disappeared whenever the horizontal lines were visible in their entirety. This observation implies that fusable contours cannot overcome the inhibitory effect triggered by rivalrous contours.

In contrast to Hochberg's findings, several other investigators have found that stereoscopic depth can survive in the face of ongoing rivalry (Blake, Westendorf, \& Overton, 1980; Kaufman, 1964; Mayhew \& Frisby, 1976; Ogle \& Wakefield, 1967; Treisman, 1962). This outcome, stereopsis taking precedence over rivalry, can be interpreted in several different ways. Processing of disparity information could occur prior to rivalry suppression or in parallel with suppression. Alternatively, disparity processing could transpire after suppression, but positional information concerning a contour could survive the inhibitory effect of suppression. In this regard, it is noteworthy that the ability to judge the location of probe targets introduced to an eye during suppression is only mildly impaired: detection thresholds are elevated only about .3 to $.5 \log$ units (e.g., Wales \& Fox, 1970), even though the rival target undergoing suppression may itself be 


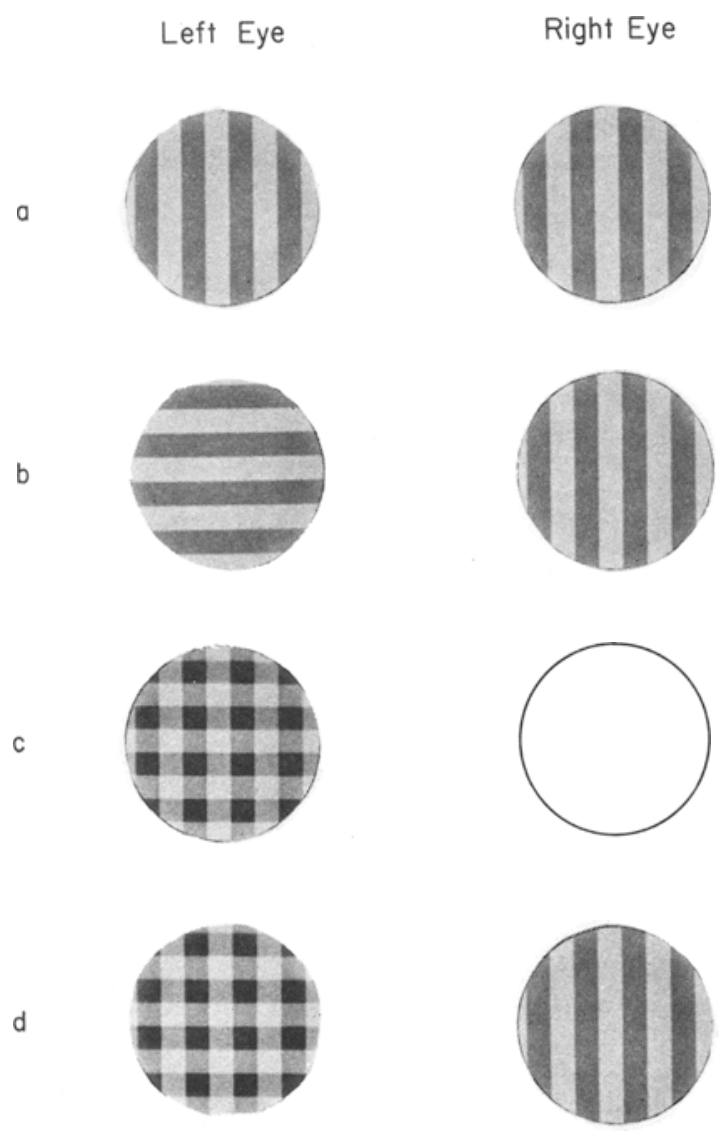

Figure 1. This series of photographs illustrates the stimulus conditions under which reaction time to contrast decrement probes was measured. Details of these conditions are described in the text. It should be noted that in our experiments rival targets were $1.2^{\circ}$ in diameter (see Figure 2b). Unlike the larger targets shown in this figure, the relatively small targets actually employed tended to yield unitary rivalry, whereby one eye's view appeared and disappeared in its entirety. Also in our experiments, strong fusion contours were included in both eyes' views (see Figure 2b); these served to stabilize binocular alignment. The targets shown in this figure lack strong fusion contours and are difficult to maintain in steady binocular alignment. Free fusion of these dichoptic pairs may not, therefore, accurately portray what observers experienced when viewing the displays in our apparatus.

several log units above threshold. Still another possibility is that local contours contributing to stereopsis are released from suppression by the presence of their fusable counterpart in the dominant eye. Of course, since these contours are compatible with those seen by the dominant eye, an observer would have great difficulty discerning a localized release from suppression of those contours.

In summary, studies of stereopsis during rivalry leave unanswered the question of which takes precedence, fusion or rivalry. In the present paper, we have employed a somewhat different strategy to tackle this question. We have used a test probe procedure to examine the fate of monocularly viewed contours that have both fusable and rivalrous counterparts contained within the other eye's view. The logic of our experiment is simple. Consider a stimulus display with the right eye (RE) viewing a vertical grating while the left eye (LE) views a vertical grating (which on its own would fuse with the RE pattern) and a horizontal grating (which on its own would rival with the RE pattern). This stimulus configuration (see Figure 1d) thus satisfies the requirements for fusion (compatible orientations) and, at the same time, provides the stimulus conditions for suppression (incompatible orientations). Now, we know that during periods of suppression the suppressed eye is significantly less responsive to stimulus changes and probes of sensitivity (Blake \& Fox, 1974; Fox \& Check, 1968; Wales \& Fox, 1970). In contrast, during genuine fusion both eyes' views are equally visible, with no indication of ongoing suppression in the form of reductions in visual sensitivity (Blake \& Camisa, 1978). Thus, sensitivity to monocular probe targets indicates whether the probed eye was in a suppressed state or not. Using this probe technique, we have measured sensitivity to changes in each component of the three-grating dichoptic display pictured in Figure 1d. Our results indicate that potentially fusable contours are exempt from suppression.

\section{METHOD}

\begin{abstract}
Apparatus
Stimuli were electronically generated on three matched CRT screens. For the LE view, two CRT displays were optically superimposed with a half-silvered mirror. On one of those CRTs, a vertical grating pattern could be displayed, and on the other a horizontal grating could be displayed. When both patterns were presented simultaneously, the LE's view resembled a grid (not a checkerboard) composed of vertical and horizontal bars. The RE viewed a third CRT on which a vertical grating could be displayed. A mirror stereoscope (see Figure 2) was used to combine the LE and RE displays. To promote unitary rivalry, each CRT screen was covered by a mask with a central circular aperture $1.2^{\circ}$ in diameter, through which the CRT raster was visible (see Figure 2). The average luminance of the display for each eye was $34 \mathrm{~cd} / \mathrm{m}^{2}$. All gratings were $5-\mathrm{c} / \mathrm{deg}$ squarewave patterns. Unless noted otherwise, contrast was 0.20 .
\end{abstract}

\section{Procedure}

While seated in a dark booth the observer looked into the stereoscope, with head held steady by a chinrest. Within easy reach of the observer's right hand was a response button, the output of which was monitored by a computer. Preceding each test condition was a $1 \mathrm{~min}$ period during which the observer simply looked at the stimulus display, to allow rivalry to become well established (Hollins, 1980). The dependent measure used to infer phenomenal suppression of a stimulus pattern was reaction time (RT) to an abrupt decrement in the contrast of one of the three grating patterns. When observation conditions were appropriate for the condition under study, the observer depressed the response button. At randomly selected times, while the button was depressed, the computer triggered an abrupt $60-\mathrm{dB}$ decrease in the contrast of one of the component gratings of the display. This lowered the contrast from 0.20 (a value at which the contours were clearly visible, at least when dominant) to .0002 (a value well below detection threshold). The observer released the response button as quickly as possible when he/she detected the disappearance of this target grating pattern. RT in mil- 
(a)
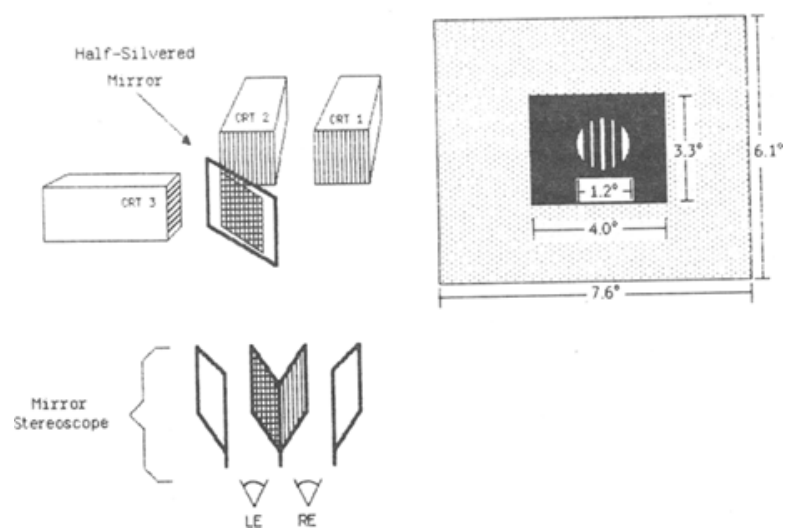

Figure 2. The dichoptic display used in these experiments. (a) The left-eye display, consisting of two CRTs whose images were superimposed via a beam splitter; the right-eye display consisted of the image from a single CRT. The left- and right-eye displays were viewed through a mirror stereoscope. (b) The angular dimensions of the display.

liseconds was measured from the onset of the contrast change to the release of the button. RTs less than $100 \mathrm{msec}$ were discarded as false alarms. Once the observer had responded to the contrast decrement, the contrast of the test grating pattern returned to 0.20 and the next trial was initiated. If the observer failed to respond to the probe within $3,000 \mathrm{msec}$, an RT of that duration was recorded, the contrast of the grating was ramped back up to 20 over $200 \mathrm{msec}$, and the computer continued with the next test trial. Before each block of trials, the observer was informed which of the component grating patterns in the display was to be the test grating (i.e., which pattern would be changing in contrast) during that next block of trials.

For the sake of clarity, procedural details for each of the test conditions are presented in the Results section.

\section{Observers}

Three observers participated in the experiment: K.H., R.B., and M.J. R.B. is one of the authors. K.H. and M.J. are experienced psychophysical observers but were naive as to the purpose of the experiment. All observers had normal or corrected-to-normal visual acuity and good stereopsis, and all reported vigorous rivalry when viewing dichoptic displays like those in Figure $1 \mathrm{~b}$.

\section{RESULTS}

Our aim was to look for any effects of binocular suppression on the individual components of the composite pattern (Figure 1d), the index of suppression being a lengthening of RT to contrast decrements of those components. Here is the rationale underlying this idea: Previous work has shown that RTs to contrast decrements in a suppressed grating are considerably longer than RTs to the same decrements occurring during dominance (Blake \& Fox, 1974). In that study, the presentation of contrast decrements was contingent on the state of rivalry reported by the observer via a response switch-the experimenter thus knew whether the probe occurred during dominance or during suppression. With the composite display em- ployed in our study, there were no obvious fluctuations in the visibility of the components. Hence, it was impossible to initiate probe trials contingent on a phenomenal state. Instead, we initiated probe trials at random times during the observation period, reasoning that if rivalry were ongoing (albeit inconspicuously) some probes would occur during dominance while others would occur during suppression. This should be reflected in the broadening of the resulting RT distribution, compared to the distribution obtained under conditions of simple fusion (Figure 1a). Before carrying out the main experiment, we performed preliminary experiments to test several questions concerning the logic of our procedure; those preliminary experiments are presented first.

\section{Are contrast decrements discriminable}

from the onset of suppression?

In simple rivalry between orthogonally oriented gratings, the transition from dominance to suppression involves a rather abrupt decrease in the perceived contrast of one of those gratings; its physical contrast, of course, remains unchanged. For our main experiment to work, observers had to be able to discriminate changes in perceived contrast occasioned by the onset of suppression from real decrements in contrast occurring during probe trials. We tested whether this was possible in the following way.

For these measurements, the LE viewed a horizontal grating and the RE viewed a vertical one (Figure $1 b$ ). Observers were instructed to track the resulting rivalry, depressing the response button when any portion of the horizontal grating was dominant and releasing it when the horizontal pattern became suppressed in its entirety. At some point from 1 to $2 \mathrm{sec}$ after the observer depressed the button (horizontal dominant), the contrast of the horizontal grating was abruptly dropped $60 \mathrm{~dB}$, rendering it invisible. These contrast decrements were presented randomly, every one to five dominance periods, so the observer could not anticipate their occurrence. Observers were instructed not to release the button when the contrast of the horizontal grating abruptly dropped. After $1 \mathrm{sec}$, the probed grating was returned to full strength, and a tone signaled the observer to resume releasing and depressing the button to track fluctuations in rivalry. The observers' success at releasing the button for fading of the horizontal grating due to rivalry suppression, but holding it down for an abrupt probe disappearance, indicated whether the observer could discriminate the probe stimulus from the onset of suppression.

A total of $\mathbf{3 0}$ trials were run in which observers were required to respond to the onset of rivalry but to ignore real contrast decrements. Observers had no difficulty distinguishing between the two conditions: among observers, the maximum number of false alarms (i.e., button releases rather than rejections of the probe presentations) was only 2 out of 30 (Observer R.B.). Furthermore, in those few cases in which false alarms were recorded, observers spontaneously reported that a probe had occurred coinci- 
dent with the onset of rivalry suppression of the horizontal grating and that they had been unable to stop their response to the rivalry alternation in time. It can safely be assumed, therefore, that contrast probes are discriminable from spontaneous fluctuations in perceived contrast. In all subsequent testing, false alarms (releases of the button in the absence of the probe) occurred on less than $10 \%$ of all trials, and in many of these cases, the button was released by the observer simply to take a break in the running of a block of trials.

\section{Does suppression lengthen $R T$ \\ to contrast decrements?}

To use RT to contrast decrements as a probe for indexing suppression, it was necessary to confirm for the conditions of our experiment that probes during suppression yield significantly longer RTs than probes during dominance. To perform these measurements, observers were again presented with the rivalrous grating pair of LE horizontal and RE vertical gratings (Figure 1b). For one condition, observers tracked the exclusive dominance of the horizontal grating by depressing the response button to indicate horizontal dominance. Every one to five buttonpresses, chosen at random, the computer would drop the contrast of the horizontal grating pattern. This test probe occurred as soon as the observer signaled horizontal dominance, to minimize the chance that a spontaneous change in rivalry state would occur prior to the probe. The observer was instructed to release the button as quickly as possible when the probe was detected. Thus, for this condition, the horizontal grating was being probed while dominant. The durations of periods of horizontal dominance when no probe was presented were also recorded, to ensure that observers were not simply making fast responses following each buttonpress.

In the other condition, observers pressed the button to indicate complete suppression of the horizontal pattern.
Every one to five buttonpresses, the contrast of the horizontal grating was dropped abruptly as soon as the signal indicating suppression was received. In this case, the horizontal grating was being probed while it was phenomenally suppressed. The observer was required to release the buton when he/she detected the change in the grating. The RTs were recorded, and the suppression durations on nonprobe intervals were also measured. Observers completed two blocks of 30 probe trials for each condition. The order of the blocks was random.

The RT distributions for probes presented during dominance and during suppression are shown in Figure 3. Note that for each observer dominance RTs cluster around 300 to $400 \mathrm{msec}$, indicating that contrast decrements are readily detected during dominance. Suppression RTs, however, are widely scattered, with the majority exceeding $700 \mathrm{msec}$. This confirms that contrast decrements are more difficult to detect during suppression. It is noteworthy that some suppression RTs, though longer than RTs during dominance, were nonetheless shorter than the suppression durations reported on concurrent nonprobe trials. This suggests that the probe, although attenuated by suppression, was still sometimes detectable.

\section{$R T$ s to contrast decrements in the composite display}

Having confirmed that contrast decrements are distinguishable from the onset of suppression and that suppression does lengthen RTs to such decrements, we were ready to proceed to the main experiment. This experiment involved measuring RTs to contrast decrements under four different display conditions: a fusion display (Figure 1a), a rivalry display (Figure $1 b$ ), a monocular LE grid (Figure 1c), and the composite display (Figure 1d).

For all conditions, the observer held the response button down and released it only upon detection of a test probe, which consisted of a $60-\mathrm{dB}$ decrement in the con-

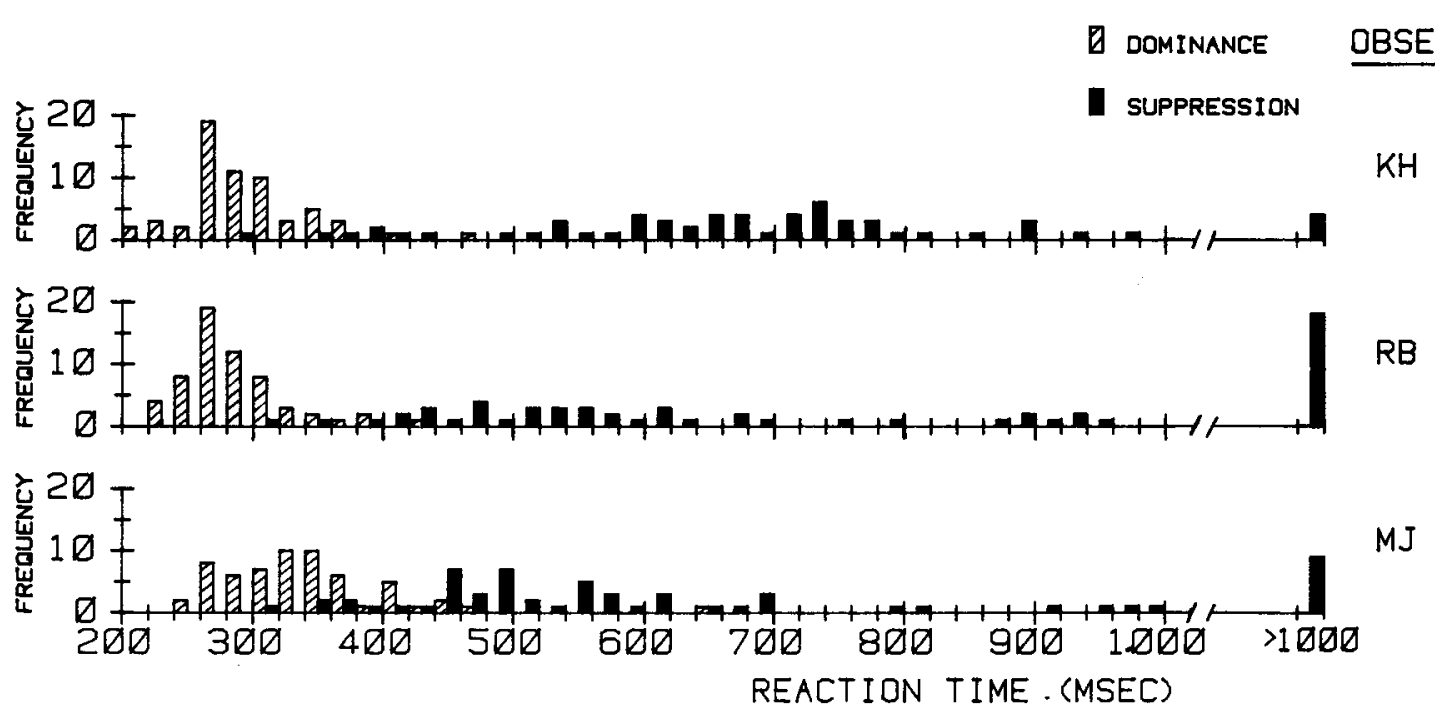

Figure 3. Histograms of reaction times to a decrement in the contrast of a grating during dominance and during suppression. Each histogram presents the results for the observer whose initials are shown at the right of the figure. 
trast of one of the gratings. Probes were presented from 3 to $8 \mathrm{sec}$ after the observer depressed the response button. Two blocks of 30 probe trials were presented under each of the following display conditions:

(1) Fusion-The RE and the LE both received vertical gratings, with the probe consisting of a decrement in the contrast of the RE grating (Figure 1a).

(2) Rivalry-The RE received vertical and the LE received horizontal, with the probe consisting of a decrement in the contrast of the vertical RE grating (Figure 1b). Observers did not track rivalry.

(3) LE grid-The RE received an unpatterned field of the same mean luminance as the grating displays, while the LE received the composite grid composed of both horizontal and vertical (Figure 1c). The probe was a contrast decrement of the LE horizontal grating.

(4-6) Composite-The RE received vertical while the LE received the composite horizontal and vertical pattern (Figure 1d). For this stimulus display, three probe conditions were tested: contrast decrement in the RE verti- cal, contrast decrement in the LE vertical and contrast decrement in the LE horizontal.

Two blocks of 30 trials were devoted to each of these six test conditions. Before each block of trials, the observer was informed which grating would be probed. Rest periods were taken whenever requested by the observer.

Raw data in the form of RT histograms for each condition are shown for one observer in Figure 4; these results are typical of all three observers. Note first that RTs under the fusion condition (top histogram) cluster around $300 \mathrm{msec}$, indicating that the drop in the contrast of the RE vertical grating was readily detected. (The removal of one member of a pair of fused gratings is occasioned by a brief, transient drop in apparent contrast; ; it was this event that observers reacted to). Contrast decrements of one component of the LE grid display were equally detectable, as evidenced by the similarity between RT histograms for the fusion condition and the LE grid condition (second panel from the top). Compare these histograms with the one associated with contrast decrements occur-
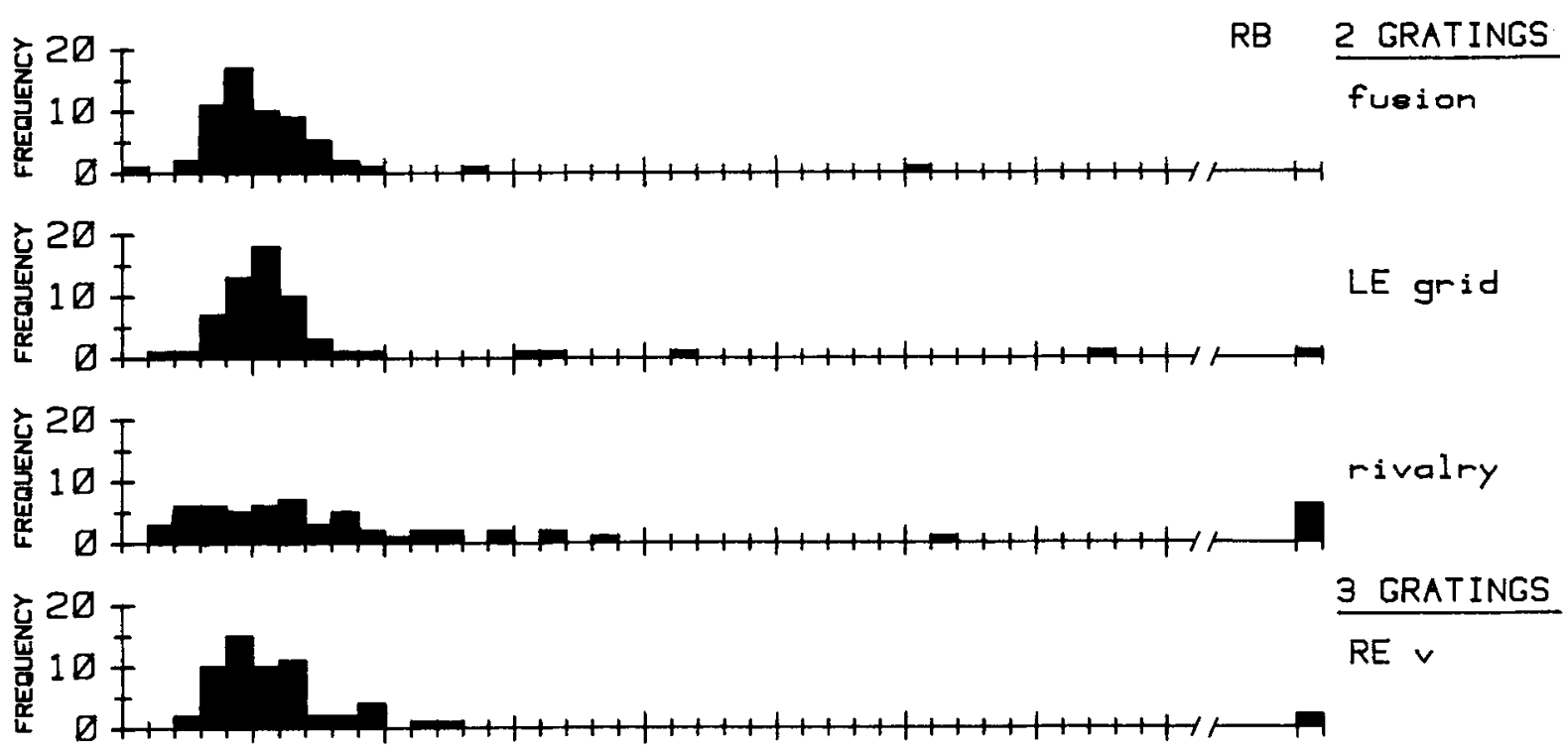

3 GRATINGS
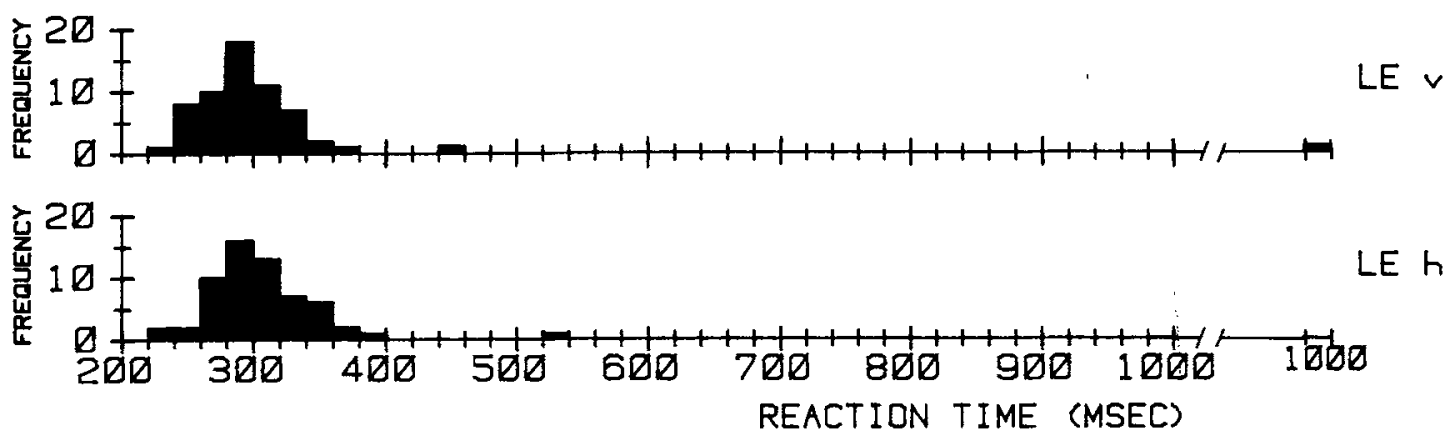

Figure 4. Histograms of reaction times to a decrement in the contrast of a grating. The "fusion" histogram consists of RTs measured during fusion (Figure 1a), the "grid" histogram of RTs measured while the observer viewed the LE grid display (Figure 1c), the "rivalry histogram" of RTs measured while the observer viewed the rival targets (Figure 1b), and the " 3 gratin" histograms of RTs measured for each component of the composite display (Figure 1d). The observer was R.B. 
ring during rivalry (third panel from the top). RTs to probes during rivalry were quite variable, with values ranging from less than $300 \mathrm{msec}$ to greater than $1 \mathrm{sec}$. In fact, for the observer shown, $10 \%$ of the RTs were $3 \mathrm{sec}$, the longest possible RT with our procedure. This wide scatter of individual RT values indicates that some probe trials (decrements in the contrast of the RE vertical grating) occurred while the RE was dominant and other probe trials occurred while the RE was suppressed. Looking now at the results for the composite display, each of the three probe conditions (RE vertical, LE vertical, and LE horizontal) yielded histograms resembling the fusion and LE grid conditions, and not the rivalry condition.

To compare these conditions statistically, the RTs for each observer were plotted in the form of cumulative frequency distributions (see Figures 5 and 6). Pairwise comparisons were made between: the fusion and rivalry RT distributions (Figure 5), the RE-vertical fusion RT distribution and the RT distribution associated with the vertical component of the composite display (Figure 6, top histogram), the LE-vertical fusion RT distribution and the RT distribution associated with the vertical component of the LE-grid display (Figure 6, middle histogram), and the LE-horizontal RT distribution associated with the LE grid display and the LE-horizontal RT distribution associated with the composite display (Figure 6, bottom histogram). (These last three comparisons, in other words, matched the distributions of RTs for each component of the composite with the counterpart distributions of RTs for the appropriate noncomposite condition.) To perform these statistical comparisons, Kolmogorov-Smirnov tests were applied to each comparison pair. This test determines the largest difference in cumulative frequency between two distributions and compares that difference (as a proportion of the total number of observations) to calculated critical values for selected type I error probabilities.

Table 1 shows the calculated statistic for each of the comparisons and the associated probability levels. As summarized in Table 1 , the rivalry RT histograms were statistically different from the fusion histograms for each observer. There was only one other case in which the difference between a pair of distributions achieved statistical significance: the LE-vertical RT histogram associated with the composite display compared with the LE-vertical fusion distribution for Observer K.H. Although we have no ready explanation for this difference, the direction of the difference is opposite that expected on the basis of binocular suppression produced by the composite display. Table 2 compares the rivalry RT histograms with the REvertical distributions and with the LE-horizontal distributions associated with the composite display. For each observer, the rivalry distributions were statistically different from the composite counterparts.

So, to summarize, RTs to contrast decrements in the composite display are equivalent to those measured under conditions of fusion, not rivalry. There is no evidence for the existence of suppression of any component of the composite display.
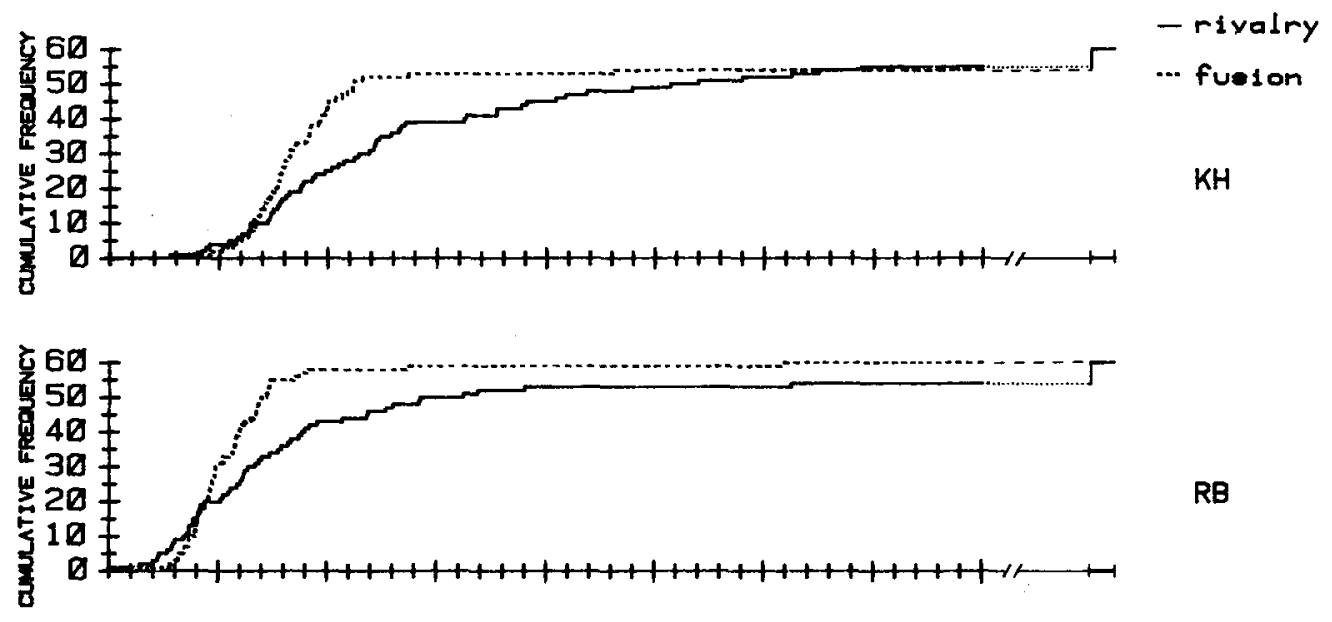

RB

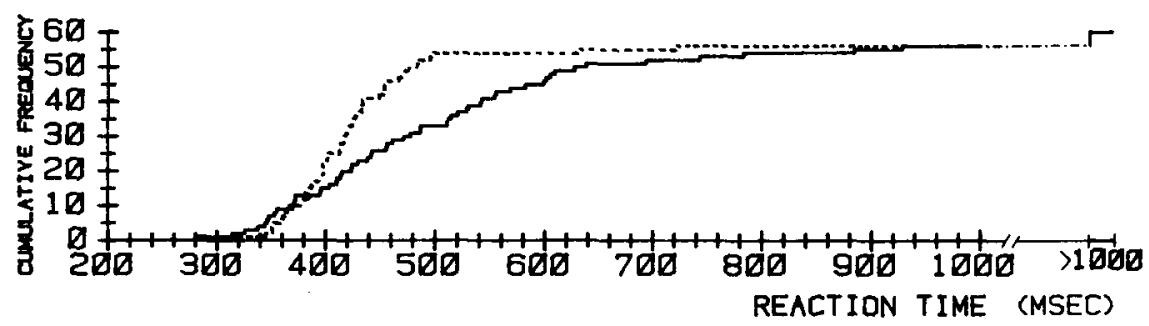

Figure 5. These three pairs of cumulative histograms compare RTs measured during fusion to RTs measured during rivalry for each of three observers. 


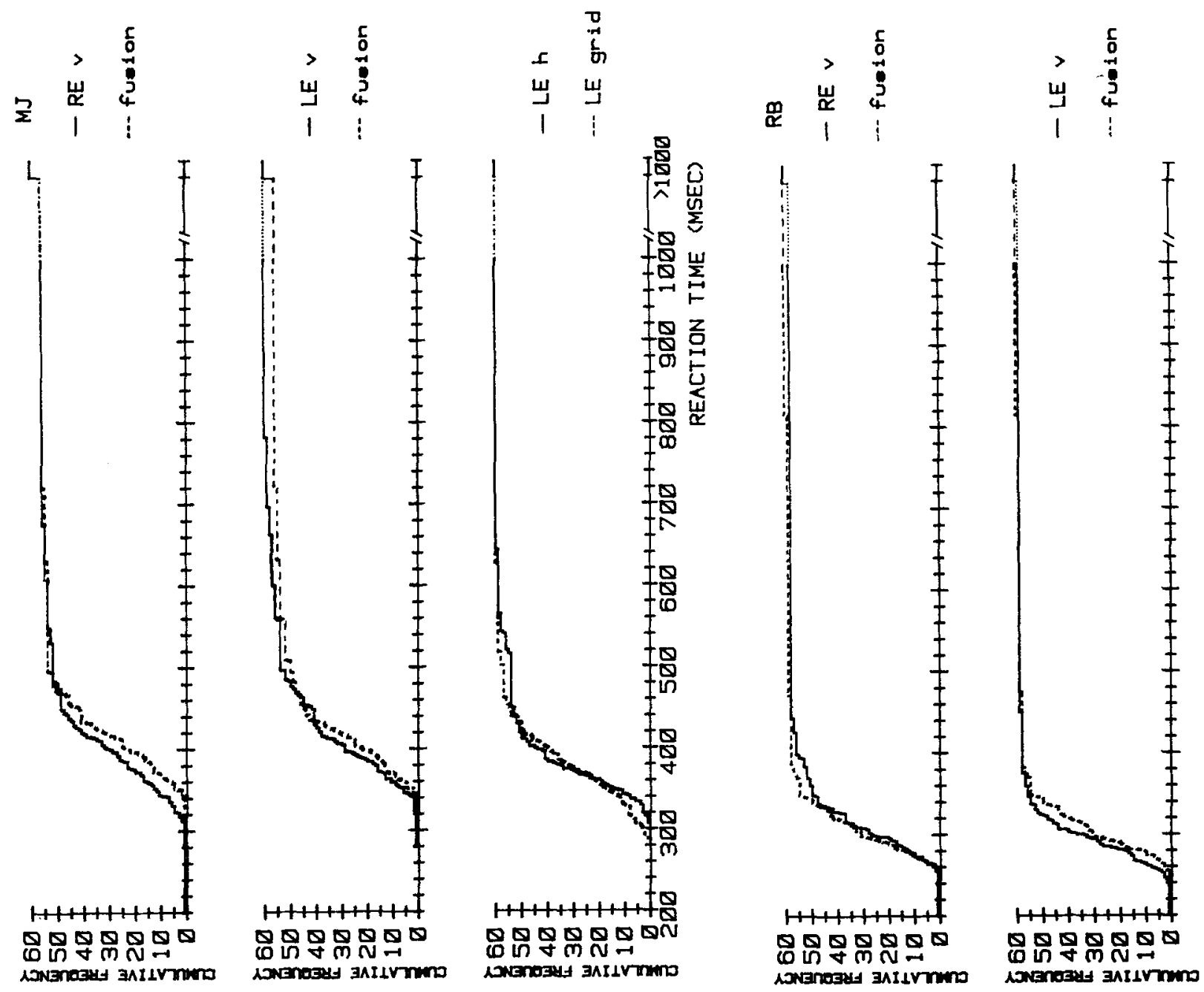



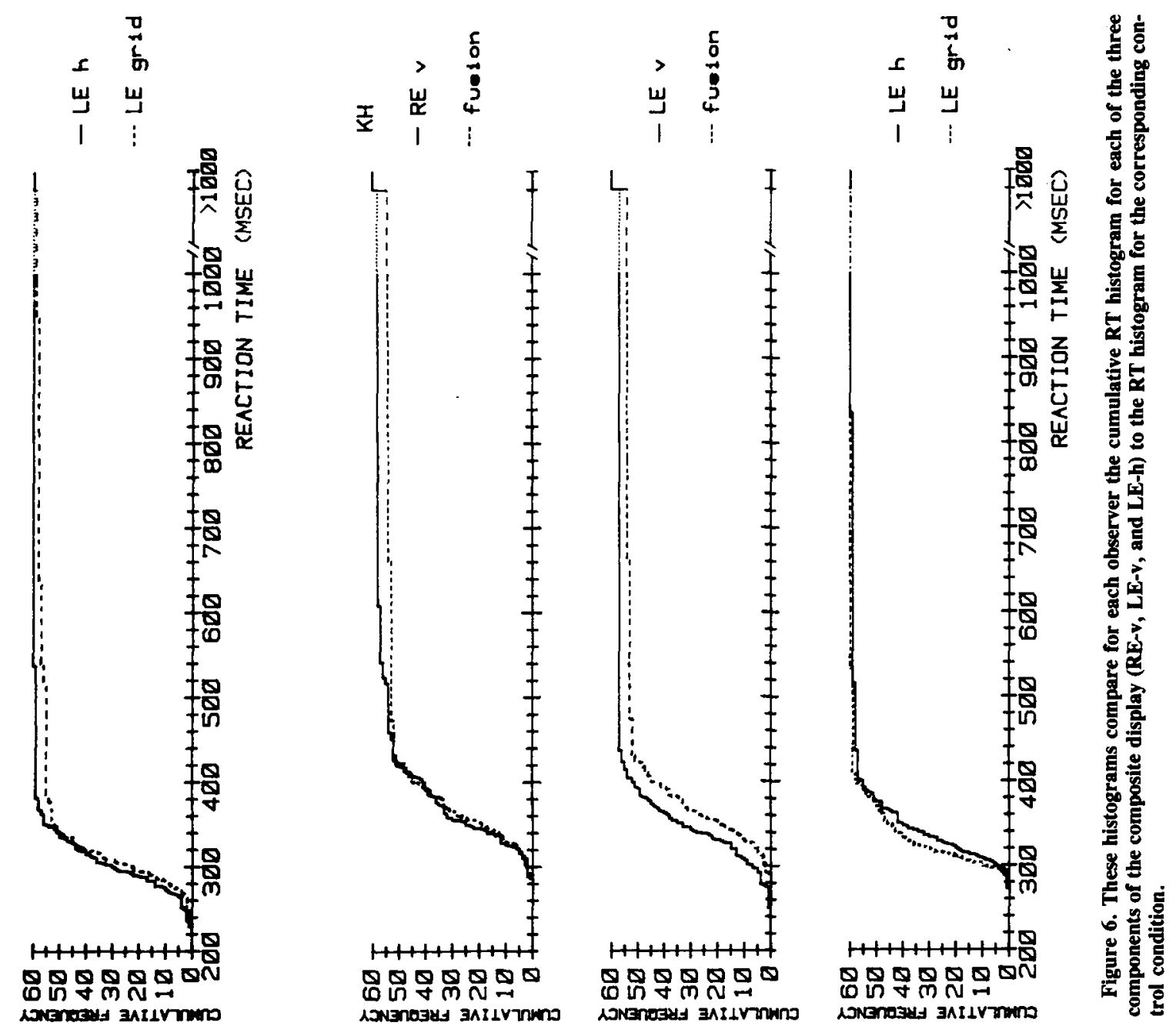
Table 1

Kolmogorov-Smirnov Comparisons of RT Distribution for Three-Grating Test Conditions and Respective Controls

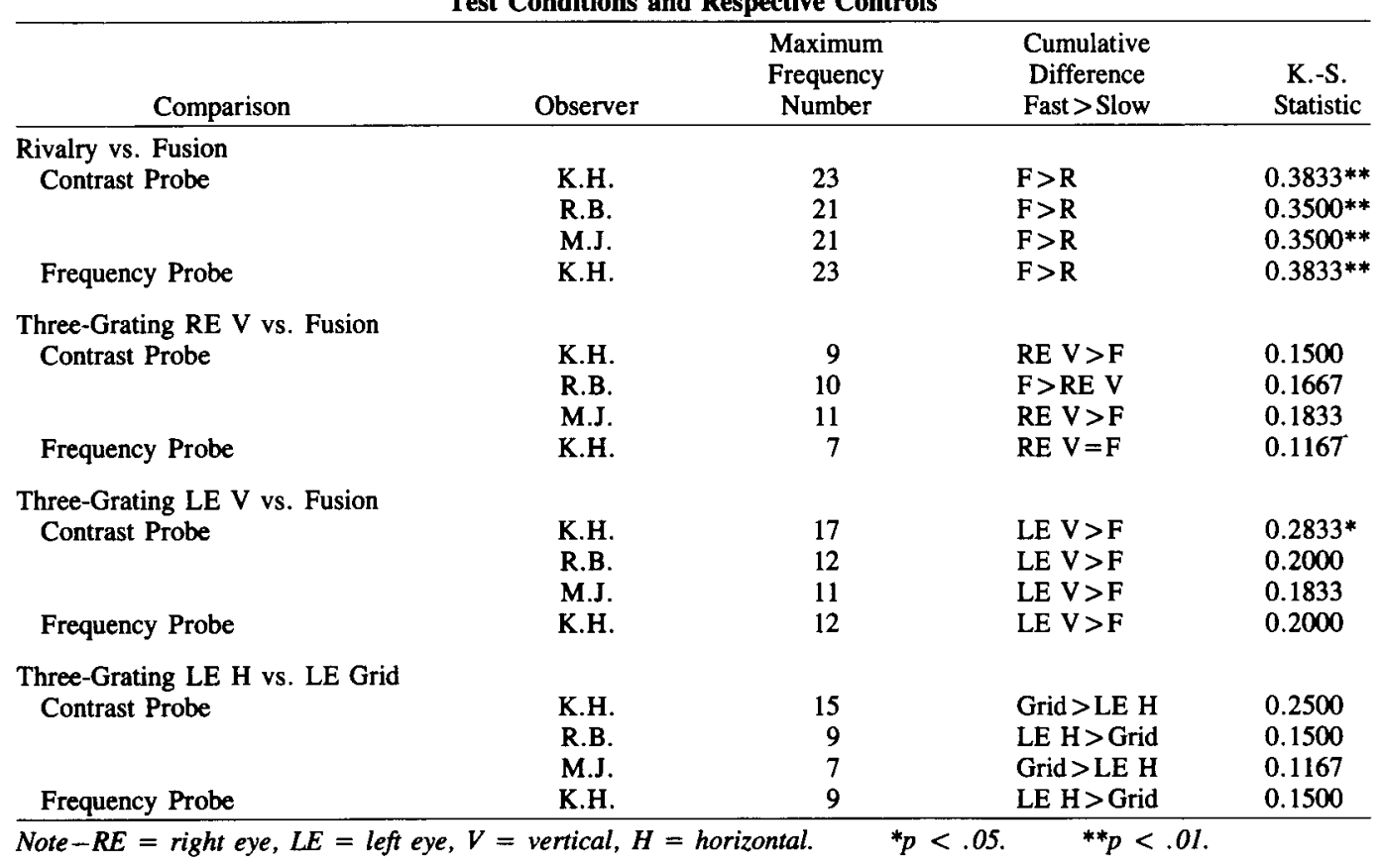

\section{$R T$ to changes in spatial frequency}

For one observer, we repeated the six conditions comprising the previous experiment, only now employing a change in spatial frequency as the test probe. We did this to insure that the pattern of results summarized in Figures 5 and 6 was not due to some artifact of using contrast decrements as a probe.

For this ancillary experiment, the RT probe consisted of change in spatial frequency from 5.0 to 7.5 cycles/deg. A spatial-frequency change this size was selected since, with a $1.2^{\circ}$ target, an even number of cycles of the grating waveform would be present before and after the frequency change; hence, overall luminance remained constant. This probe was quite distinctive and in no way resembled fluctuations in rivalry. All procedures were exactly the same as those used with the contrast probe, with two blocks of 30 RTs collected for each of six conditions.

Results for this condition replicated the contrast probe findings in every way; the entries labeled "frequency probe" in Table 1 show the Kolmogorov-Smirnov statistics for the relevant comparisons in this experiment. Again, only the rivalry/fusion distributions were significantly different.

\section{DISCUSSION}

These results provide no evidence for suppression (i.e., a lengthening of RT) of any component within either eye's

Table 2

Kolmogorov-Smirnov Comparisons of RT Distributions for Three-Grating Test Conditions and Rivalry

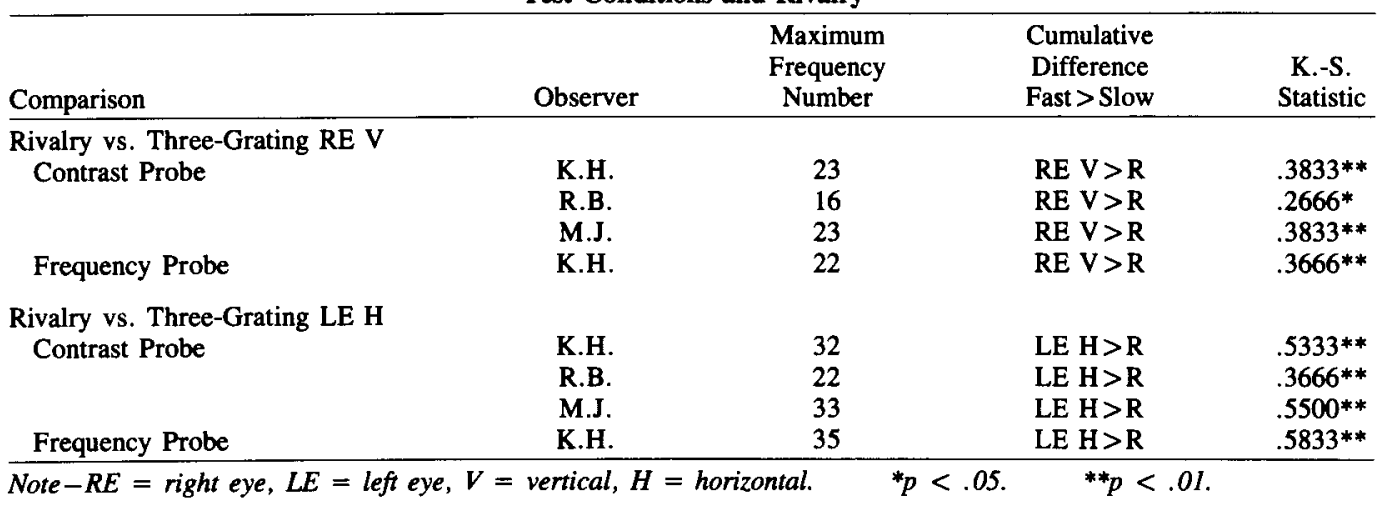


view of the display pictured in Figure 1d. This finding also squares with observers' descriptions of the subjective appearance of that display. When queried about any apparent fading of grating components, two observers reported seeing no fluctuations in the visibility of either horizontal or vertical. The third observer said the apparent contrast of the horizontal grating (seen by the left eye) did occasionally wax and wane, but at no time did that grating completely vanish as it does during rivalry. Instead, these occasional fluctuations in the apparent contrast of the horizontal pattern resembled the variations in apparent contrast experienced when viewing the LE-grid pattern (Figure 1c); this phenomenon is probably monocular rivalry (e.g., Wade, 1975).

From these results, we conclude that the presence of matching features in the two eyes' views stabilizes the binocular percept. Evidently, the binocular visual system first seeks to establish correspondence between image features contained in the two monocular views. Failure to establish such correspondence leads to binocular suppression, an effective means for eliminating diplopia and/or confusion. When correspondence is established, however, binocular fusion takes precedence over suppression. Only those monocular features with no interocular counterpart participate in the rivalry process. According to this scheme, the LE and RE vertical components of the composite display would constitute a binocular match (and hence be exempt from binocular suppression). In the absence of a corresponding RE feature, the LE horizontal component would be subject to rivalry. However, since the RE contained no unmatched features, the LE horizontal contours would represent the only input to this rivalry process. It is well established that when a monocular contour is paired dichoptically with a uniform field, the contour dominates almost continuously (e.g., Asher, 1953; Blake \& Camisa, 1978). Hence, the horizontal component would be visible continuously along with the matched, vertical components. Had the other eye's view contained remaining unmatched features, we would expect the horizontal contours to rival with those features.

Our conclusion is consistent with the dual process model of binocular vision proposed by Julesz and Tyler (1976). They posited the existence of a rivalry mechanism (stimulated by uncorrelated monocular input) and a fusion mechanism (stimulated by correlated monocular input). Based on observers' abilities to detect transitions from correlation to uncorrelation (or vice versa) in random-dot stereograms, Julesz and Tyler concluded that the fusion mechanism exerted a strong inhibitory influence over the rivalry mechanism. This conclusion, of course, dovetails nicely with our finding that potentially fusable contours are exempt from suppression. Julesz and Tyler also found that observers required between 17 and $53 \mathrm{msec}$ to detect transitions from an uncorrelated (i.e., rivalrous) state to a correlated (i.e., fusable) state. This may explain why a brief probe target presented to an eye during rivalry suppression does not combine with a corresponding probe presented to the dominant eye to fully enhance binocular detection performance (Westendorf, Blake, Sloane, \& Chambers, 1982).

The conclusion that binocularly matched contours are exempt from suppression sheds a different light on how we think about the relation between stereopsis and rivalry. Previously, we thought that disparity information was processed prior to suppression or in parallel with the rivalry mechanism (Blake, Westendorf, \& Overton, 1980). Perhaps, though, the addition of disparate contours to one eye liberates from suppression the corresponding contours seen by the other eye, and once this correspondence has been established, disparity processing proceeds. It must be noted, however, that Hochberg's (1964) observations remain inexplicable even within this context.

In summary, the current results provide no support for a strict suppression theory in which contours seen by one eye suppress contours seen by the other regardless of the similarity of those contours (e.g., Makous \& Sanders, 1978). Instead, our findings imply that fusion and rivalry are separate processes that interact in a way such that fusion takes precedence over rivalry. This precedence would insure that binocularly matched image features are registered, despite other incongruities in the two eyes' views which might trigger suppression. According to this view, binocular single vision involves an interplay between fusion and suppression (see also Cogan, 1982), with rivalry operating only on those monocular features lacking interocular counterparts.

After our paper was accepted for publication, we learned that O'Shea and Dodwell (unpublished manuscript) had performed similar experiments, which lead them to the same conclusion as ours; we thank them for sharing their findings with us.

\section{REFERENCES}

ASHER, H. (1953). Suppression theory of binocular vision. British Journal of Ophthalmology, 37, 37-49.

BLAKE, R., \& CAMISA, J. (1978). Is binocular vision always monocular? Science, 200, 1497-1499.

BLAKE, R., \& Fox, R. (1973). The psychophysical inquiry into binocular summation. Perception \& Psychophysics, 14, 161-185.

BlaKe, R., Fox, R. (1974). Binocular rivalry suppression: Insensitive to spatial frequency and orientation change. Vision Research, 18, 541-544.

Blake, R., Westendorf, D. H., \& Overton, R. (1980). What is suppressed during binocular rivalry? Perception, 9, 223-231.

Breese, B. B. (1899). On inhibition. Psychological Monograph, 3, Whole No. 11 .

Cogan, A. (1982). Monocular sensitivity during binocular viewing. Vision Research, 22, 1-16.

Fox, R., \& CHECK, R. (1968). Detection of motion during binocular rivalry suppression. Joumal of Experimental Psychology, 78, 388-395.

HochBERG, J. (1964). Depth perception loss with local monocular suppression: A problem in the explanation of stereopsis. Science, 145, 1334-1336.

Hollins, M. (1980). The effect of contrast on the completeness of binocular rivalry suppression, Perception \& Psychophysics, 27, 550-556.

JONES, R. K., \& LEE, D. N. (1981). Why two eyes are better than one The two views of binoculat vision. Joumal of Experimental Psychology: Human Perception and Performance, 7, 30-40. 
JULESZ, B., \& TYLER, C. W. (1976). Neurontropy, an entropy-like measure of neural correlation, in binocular fusion and rivalry. Biological Cybernetics, 23, 25-32.

KaUfMaN, L. (1964). Suppression and fusion in viewing complex stereograms. American Journal of Psychology, 77, 193-205.

Makous, W., \& SANDERS, R. K. (1978). Suppressive interactions between fused patterns. In J. C. Armington, J. Krauskopf, \& B. W. Wooten (Eds.), Visual psychophysics and physiology. New York: Academic Press.

MAYhew, J. E. W., \& FrisBy, J. P. (1976). Rivalrous texture stereograms. Nature, 264, 53-56.

NELSON, J. I. (1975). Globality and stereoscopic fusion in binocular vision. Journal of Theoretical Biology, 49, 1-88.

OGLE, K. N. (1964), Researches in binocular vision. New York: Hafner.

OGle, K. N., WAKefield, J. M. (1967). Stereoscopic depth and binocular rivalry. Vision Research, 7, 89-98.

Ono, H., ANGUS, R., \& Gregor, P. (1977). Binocular single vision achieved by fusion and suppression. Perception \& Psychophysics, 21, 513-521.

Treisman, A. (1962). Binocular rivalry and stereoscopic depth perception. Quarterly Journal of Psychology, 14, 23-37.

WADE, N. J. (1975). Monocular and binocular rivalry between contours. Perception, 4, 85-95.

WALES, R., \& Fox, R. (1970). Increment detection thresholds during binocular rivalry. Perception \& Psychophysics, 15, 61-65.

Westendorf, D. H., Blake, R., Sloane, M., \& Chambers, D. (1982). Binocular summation occurs during interocular suppression. Journal of Experimental Psychology: Human Perception and Performance, 8, 81-90.

(Manuscript received October 22, 1984; revision accepted for publication January 2, 1985.) 